\title{
Personal Life Stories: Common Deviations from the Cultural Life Script
}

\author{
Sharda Umanath ${ }^{1} \&$ Dorthe Berntsen ${ }^{2}$ \\ ${ }^{1}$ Duke University, ${ }^{2}$ Center on Autobiographical Memory Research, Aarhus University
}

This is an Author's Accepted Manuscript of an article published in Nordic Psychology, Volume 65 , Issue 2, 2013, available online at: $h$ ttp://www.tandfonline.com/10.1080/19012276.2013.807662. Changes resulting from the publishing process, such as peer review, editing, corrections, structural formatting, and other quality control mechanisms may not be reflected in this document. Changes may have been made to this work since it was submitted for publication. 


\begin{abstract}
Autobiographical memories are typically thought of as people's memories for personal life events. Yet, life stories do not exist in isolation; they are shaped by the shared social norms and prescriptions of one's culture as to the order and timing of important transitional events: a cultural life script. An individual's knowledge of their culture's standard life script does not arise from compiled individual life events but is learned detached from particular personal experiences. When probed, many people's most important personal life events do not match the cultural life script exactly. We note that even some commonly experienced life story events do not match the life script and that their qualitative differences have not been systematically investigated. Why are some common life story events in the cultural life script while others are not? To begin exploring these differences, we examined what distinguishes two main types of commonly important events within people's personal life stories: events that do overlap with what they conceive of as their culture's life script and events that do not. We offer a secondary data analysis of the Rubin, Berntsen, \& Hutson (2009) life story data, exploring American life story data using the proposed categories of events, the various ratings the authors previously collected, and unused demographic information of interest. Given that this is simply a first step in characterizing the nature of common important life story events, we also provide some speculation for future avenues of investigation and the broader relevance of this work. (247 words)
\end{abstract}




\section{Personal Life Stories: Common Deviations from the Cultural Life Script}

With the level of cross-cultural communication only increasing, knowledge and understanding of the diversity between and within cultures becomes ever more important for building amiable and mutually-beneficial relationships. This kind of research can be informative for immigration, for internal conflicts cultural groups experience, and for a variety of other issues we face today. One way to begin empirically understanding the effects of diversity on people's actions and conceptions of their own lives is to investigate this diversity within a given culture. Here, we examine how people's personal life story memories agree with or deviate from their cultural life script - that is, their culturally shared expectations as to the order and timing of important transitional events (Berntsen \& Rubin, 2004). We are especially interested in examining shared themes among memories that deviate from the script in order to see if people's remembered lives have more in common than what the life script prescribes. We first briefly describe the notion of autobiographical memory. We next discuss the relation between personal life story memories and cultural life scripts. Finally, we present our analyses of the correspondence between life stories memories and the cultural life script in an American sample of university students as a first step towards understanding these issues.

\section{Autobiographical Memory: Remembered Lives}

Broadly, when one thinks of "memory," one may generate a variety of different ideas:

One could remember having lunch with a friend yesterday, that plants take in carbon dioxide and release oxygen, or one's $12^{\text {th }}$ birthday party. Cognitive psychologists delineate such 
memories into differential categories. Memories for general knowledge and facts about the world, such as factoids about the way plants work, tend to be devoid of connections to particular instances of learning; these sorts of memories are referred to as semantic memories. Memories for particular events, such as a lunch one had yesterday or one's $12^{\text {th }}$ birthday party, tend to include details such as the location, the time, and the mood one was in, and are called episodic or autobiographical memories. But autobiographical memories can also be more abstract in nature; that is, they can refer to experiences that are not tied to a specific time, such as remembering what it was generally like to attend school parties while growing up. While there are a number of types of memories that cognitive psychologists distinguish, here we will consider the relationships among these particular types of memory.

As mentioned above, one way that memories for specific events (episodic memories) are typically distinguished from those for facts (semantic memories) is by their rich contextual details. A possible relationship between the two is that semantic memories are formed from compiled episodic memories as contextual details (e.g., source) are forgotten over time (e.g., Tulving, 1985). This type of relationship between episodic and semantic memories has been investigated in a broad literature in terms of learning episodes and general knowledge. However, the interplay between episodic and semantic memories has not been as comprehensively examined within autobiographical memory, especially within personally important events.

What we typically think of as autobiographical memories are episodic: people's memories for personal life events (life story events). Yet life stories do not exist in a vacuum, 
developing only from specific personal experiences; they are also shaped by the shared norms and prescriptions of one's culture (Conway \& Bekerian, 1987; Lubrosky, 1993; Settersten \& Hagestad, 1996). As a result, people share an idea of the prototypical life, a cultural life script (Berntsen \& Rubin, 2004; see also cultural concept of biography in Habermas \& Bluck, 2000; for other names for the cultural life script, see McAdams, 2001). A cultural life script is organized by society's dictates and expectations of a certain idealized order and timing of a variety of life events (Collins, Pillemer, Ivcevic, \& Gooze, 2007; Neugarten, Moore, \& Lowe, 1965). In turn, cultural life scripts are thought to regulate behaviors across societies (Erdoğan, Baran, Avlar, Tas, \& Tekcan, 2008; Settersten \& Hagestad, 1996; Rubin \& Berntsen, 2003; see also Zaragoza Scherman in this issue), as well as influence people's memories for and recall of their life stories (Bohn, 2010; Rubin \& Berntsen, 2003; Thomsen \& Berntsen, 2008).

One can argue that such cultural life scripts involve a form of cultural learning (Tomasello, 2001) and are semantic knowledge. Even young people who have not experienced many life script events still know their culture's life script (Berntsen \& Rubin, 2002; 2004; Bohn \& Berntsen, 2011; Janssen \& Rubin, 2011), with this knowledge increasing in normativity into adolescence (Bohn \& Berntsen, 2008; Neugarten et al., 1965). Thus, unlike some episodic and semantic memories for information, it appears that life scripts are not formed from compiled individual life events but are instead learned detached from specific personal experiences (Bohn, 2010; Rubin \& Berntsen, 2003). 


\section{The Relationship between Personal Life Stories and Cultural Life Scripts}

Imagine that you are asked to think about important life events. If you were to come up with the seven most important events in your life, which events would you nominate? Then, you are asked to imagine an ordinary newborn infant and what his/her life will be like. Which seven events would you nominate as the most important events that will occur through the course of this newborn's completely prototypical life ahead? Both of these tasks are likely to be easy to complete, taking little mental effort to come up with personal and typical important life events. Thinking about the two lists of events, you might speculate that they would have some overlap, but that the two lists would not be identical. That is, you might list some events only as personally important life events, while you might select others solely for the more generic important life events list.

Rubin, Berntsen, and Hutson (2009) studied the overlap between the events that people nominated as the seven most important events in their own lives and in the life of a hypothetical person living a completely ordinary (prototypical) life. Their participants were 100 Duke undergraduate students, with a mean age of 18.7 years. For the personal life events, the participants were asked to imagine that they were to tell their life story to a new friend, whom they had just met and who therefore did not know anything about their past. They were asked to imagine telling their personal stories to this (fictitious) friend with whom they were absolutely confident and with whom they could be completely honest. Their task was to note the seven memories of events from their own personal lives - from their birth to their present age - that they thought were most central to their life story. For the life script task, on the 
other hand, the instruction was the following: "Imagine a quite ordinary infant (choose according to your own gender). It cannot be a specific infant that you know, but a prototypical infant in our culture with a quite ordinary life course ahead. Your task is to write down the seven most important events that you imagine are most likely to take place in this prototypical infant's life, from birth to death."

Compiled across participants, the latter is a conceivably empirical measure of life events that a given population considers important, without researcher bias; individuals are not constrained by experimenter conceptions of what particular events are important. The cultural life script has been operationalized as those events which four or more individuals (out of 100) nominate as among the seven most important events that are likely to occur in a typical newborn's life course (e.g. Bohn, 2010; Bohn \& Berntsen, 2008, 2011; Berntsen \& Rubin, 2004; Erdogan et al., 2008; Janssen \& Rubin, 2011; Rubin et al., 2009). Of note is that one's personal life events do not typically match one's cultural life script exactly, though it is used as a structure for organizing and recalling life story memories (Berntsen \& Rubin, 2004; Rubin et al., 2009; Bohn, 2010). Having collected the first set of such data from Americans, the authors found less overlap between life script events and personal life story events in Americans (46\%) as compared to Danes (70\%). That is, only $46 \%$ of what Americans nominated as the most important events in a typical person's life overlapped with what they listed as the most important events in their own lives. The Danes, in contrast, showed a great deal more overlap (70\%) between what they believed were the most important events in a typical person's life and in their own lives. Citing greater diversity in American culture as the possible reason for this 
large difference, the authors did not add further to that explanation. We discuss this finding in greater detail later on.

Beyond distinguishing life scripts and life stories as mentioned above, what qualitatively characterizes the similarities and differences between personal life stories and cultural life scripts has not been empirically investigated. Whereas it is contextual details that seem to be forgotten or discarded in the transition from episodic to semantic memory for information, the qualitative characteristics that differentiate personal life stories and cultural life scripts are unknown. We propose one way of broaching this question through the specific examination of what distinguishes two types of important events within people's personal life stories: ones that do overlap with what they conceive of as their culture's life script and ones that do not.

\section{Common Life Stories}

Based on the standard collection of life story and life script event data as described above, life story events, those that people nominate as the most important in their own lives, can be broadly categorized according to their overlap (or lack thereof) with the generated cultural life script of a relevant group (see Table 1). We have all experienced completely unique events that we might describe as among the most important of our lives (e.g., volunteering at the Olympics), and it comes as no surprise that these wholly distinctive events do not overlap with one's culture's life script. However, there do exist "common" life story events, which many people may have experienced (e.g., moving to a new city; see Table 2 for the list of commonly experienced life story events from Rubin et al., 2009). Here, we define the commonness of life stories across people in the same way as the cultural life script of a group is operationalized: for 
a life story event to be "common," four or more individuals (out of 100) must nominate it as among the seven most important events they have personally experienced. All further discussion referring to life stories focuses on these commonly experienced life story events; Table 2 displays the commonly nominated life story events from Rubin et al. (2009) along with their frequency of nomination in the life script task and the life story task.

Though such events are widely experienced, only some "common" life stories overlap with the cultural life script, whereas others are never suggested as life script events by even a single individual. In an effort to quantify the degree of overlap that a common life story has with a group's cultural life script, one can create three categories of event types (See Table 1 for examples of each type). The first category of common life story events consists of those events that are in the group's cultural life script. That is, these events are the ones in the cultural life script (CLS events) that overlap with people's most important life story events. One can then consider those events that are not in the group's cultural life script (Non-CLS events): These are the common life story events that were nominated by fewer than four people in the life script task. Conceptually, this grouping lumps together those events that were occasionally nominated as life script events (but less than four times) and the events that were never nominated as life script events by any individual. Therefore, a natural third category consists exclusively of the commonly experienced life story events that no one ever nominated as among the most important events in a typical newborn's life course (Unique Life Story events). That is, the Unique Life Story events conceptually consist of a subset of the Non-CLS eventsthose that were never nominated in the cultural life script task. However, for the empirical 
analyses that follow, these two categories of events have been completely separated: Non-CLS events represent the common life story events which participants did occasionally nominate as life script events (i.e. life story events that were listed as life script events 1-4 times); Unique Life Story events refer to the common life story events that were never nominated as among the most important events in a typical newborn's projected life. Creating and applying these categories to life story data can afford us a clearer understanding of qualitatively what sorts of events fall into each one (see Table 1).

\section{Secondary Data Analyses}

As a first step to understanding differences among the categorizations of common life stories discussed above, we conducted a secondary data analysis of the Rubin et al. (2009) life story data, applying these event types. This dataset includes responses from 100 Duke undergraduates, including 55 females and an age range from 18 to 28 , as well as 111 Danish undergraduates from Aarhus University, including 92 females and an age range from 21 to 49 . Our secondary data analyses focused exclusively on the American students. Each participant nominated the seven most important events in his or her own life and in a prototypical ordinary life. For both types of event nominations, participants rated how common the event is in people's lives generally (prevalence), how important the event is (importance), at what age people would typically experience it (age), and how emotionally positive or negative the event is (valence). Prevalence ratings were made on a scale of 1 to 100 . The importance ratings were made on a scale of 1 to 7 . Valence was rated on a scale from -3 to +3 with -3 being highly 
negative and +3 being highly positive. The American participants were also asked whether or not they were native speakers of English.

In terms of commonly experienced life story event nominations, overall, participants listed approximately 583 common life story events and 117 events that they did not share with at least three other people. Thus, on average, a given participant nominated 5.83 life story events that were shared with at least three other people and 1.17 events that were not. There were no participants who did not nominate any common life story events.

In the secondary data analyses that follow, all results, unless otherwise stated, were significant at the .05 alpha level. A Geisser-Greenhouse correction was used for violations of the sphericity assumption of ANOVA.

\section{Differences in Life Story Phenomenology based on Life Story-Life Script Overlap}

We conducted a series of repeated measures ANOVAs to examine any differences in the various ratings collected in Rubin et al. (2009) based on the event categories in the personal life stories. In terms of the sheer number of common events nominated as important personal life story events, we found that participants nominated the event types at different rates, $F(2,198)$ $=88.84, M S E=1.70, \eta_{\mathrm{p}}{ }^{2}=.47$ (see Table 3). First, participants nominated significantly more CLS events $(M=3.18)$ than Unique Life Story events $[M=1.58 ; t(1,99)=7.50$, SEM $=.21]$. The high number of CLS event nominations indicates the validity of the life script task as tapping those events that are experienced broadly by a group of people within a given culture (e.g. Collins et al., 2007) and supports Berntsen \& Rubin's (2004) claim that the CLS is used to process and organize life stories (see also, Clarke, 1995). That is, CLS events are nominated in the cultural 
life script so often for a reason: They truly are experienced and remembered as central to the life story, and thus are nominated as important personal life events more than other events in life. This finding is corroborated by participants' ratings of the prevalence of these event types: Participants demonstrated an accurate awareness of how prevalent these different types of events typically are in people's lives (see Table 3), matching the trend of commonality seen in Table 2, $\left[F(2,92)=7.84, M S E=973.04, \eta_{\mathrm{p}}{ }^{2}=.15\right]$. They rated CLS events as significantly more prevalent in people's lives $(M=64.43)$ than either Non-CLS events $[M=45.96 ; t(1,53)=4.42$, SEM $=4.52]$ or Unique Life Story events $[M=39.99 ; t(1,83)=7.49, \operatorname{SEM}=4.26]$.

Second, both CLS events and Unique Life Story events were nominated more often than Non-CLS events $[M=.77 ; t(1,99)=13.44, \operatorname{SEM}=.18$ and $t(1,99)=-5.25, \operatorname{SEM}=.15$, respectively]. This suggests that among common life story events, events that are unique to people's life stories (and are never implicated as part of the life script) stand out more in people's minds as key personal events as compared to events that seem to be more commonly shared (and are tentatively nominated as being part of the life script). Speculatively, this may be because the former play a more distinctive and salient role for life story and identity, especially in an individualistic culture.

Interestingly, participants did not rate the importance of events differently based on the type of event, $F<1$. The pattern of the means for each event type shows a trend suggesting that participants rated CLS events as more important $(M=6.07)$ than either Non-CLS ( $M=5.87$ ) or Unique Life Story events $(M=5.78)$. Thus, the data show a hint that events that overlapped with the cultural life script were considered mildly more important than those events that did 
not. However, given that this pattern was not significant, it seems that commonly experienced life story events are considered equally important in participants' minds, regardless of how much or how little they overlap with the cultural life script.

Finally, we found evidence for differences in valence ratings across these event categories, $F(2,92)=4.10, M S E=1.91, \eta_{\mathrm{p}}{ }^{2}=.08$ (see Table 3). Follow-up $t$-tests revealed that participants rated Unique Life Story events $(M=1.70)$ as significantly more positive than NonCLS events $[M=.88, t(46)=-2.40, S E M=.34]$ whereas CLS events were valenced no differently than either of the other event types $(M=1.36)$. This was surprising as Berntsen and Rubin (2004) postulated that events that are part of the cultural life script are typically considered to be more positive than other life events. Thus, we expected that CLS events would be rated more positively than the other event-types. Furthermore, contrary to the idea that deviations from the cultural life script might represent problems, unhappiness, or pursuits that did not work out in one's life (Rubin \& Berntsen, 2003), in this case, events that are uniquely part of people's life stories do not appear to be negatively valenced and are, in fact, the most positively rated. One possible explanation is that the events people commonly choose to nominate that are not part of the cultural life script are events that were remembered as positive instances of having exerted control over one's life (e.g., entering a long-term relationship or having an epiphany; Gluck \& Bluck, 2007). It could also be the case that it is uncommon life story events that fit the postulated description of deviations from the cultural life script. Another possible explanation might be the population; it is possible that populations with more stressful events in their past (than college students at Duke University are most likely to have) would show 
more valence related differences between life script events and other events. This is supported in a study of considerably older Danish university students (mean age $=26.5$ years) in which important autobiographical memories that did not correspond to the life script more frequently referred to stressful events than those that did correspond (Berntsen \& Bohn, 2010).

These secondary data analyses revealed differences in the sheer number of nominated life story events, ratings of commonality, ratings of importance, and ratings of valence based on whether commonly experienced events are part of the cultural life script, sometimes considered part of the cultural life script or unique to personal life stories. Such evidence is just the beginning for our understanding of the relationship between life story and cultural life script events.

\section{Cultural Influences on Life Story-Life Script Deviations: The Role of Native Language}

As mentioned above, Rubin et al. (2009) suggested that cultural diversity was the likely cause of Americans having much less overlap between their personal life stories and the American cultural life script (compared to Danish life stories with the Danish life script). Turning that idea on its head, we hypothesized that focusing on the deviation of life story events from life script events may be an informative approach. One possibility is that a person's individual deviations from events that he/she believes are typically most important in life indicate that not only is one living a different life than the culturally-prescribed and idealized one, but is also aware that he/she is doing so. Perhaps the deviations indirectly express one's identification (or lack thereof) with the culture in which one lives and the nature of that identification. We can consider the Unique Life Story events as "common" deviations from the life script. To explore if 
such a possibility is credible, we analyzed differences among these common life story event categories based on a demographic factor: native speaking of English.

Twenty-one percent of the American dataset on life stories and the American cultural life script includes participants who were not native speakers of English. Though they collected the information, Rubin et al. (2009) did not investigate specific differences based on native speaking of English. While not a perfect measure of one's inclusion in a society, calling a language one's native tongue does suggest some form of identification with the associated culture. The fact that English is not one's native language could certainly affect that individual's conception of the most important events in his or her own life as well as in that of an ordinary newborn in American society.

Using a 2 (Native Speaker of English: Yes, No) X 3 (Event Type: CLS, Non-CLS, Unique Life Story) repeated measures ANOVA, we explored the effect of native speaking of English on the various ratings that participants provided for each event. In terms of the number of events that participants nominated, native and non-native speakers of English differentially nominated events within each event category. While interaction between native speaking of English and life story event type was not significant $\left[F(2,196)=2.34, M S E=1.67, \eta_{p}{ }^{2}=.023, p=.10\right]$, we suspected that this may be due to the small number of non-native speakers on English in the sample. Furthermore, we specifically expected that native and non-native speakers of English might differ on the number of Unique Life Story events they nominated (see Figure 1 for the number of events nominated for each event category). In a planned comparison, we found that non-native speakers nominated significantly more common life story events that occurred 
uniquely in life stories than native speakers of English $[M=2.05$ v. 1.46; $\mathrm{t}(98)=2.17, \operatorname{SED}=.27]$. This result lends some credence to the idea that deviation from the standard culture of the society in which one lives can be indicative of cultural diversity; one must keep in mind, though, that the Unique Life Story events are still commonly experienced. One possibility is that these non-native speakers of English are adhering to another cultural life script or a sub-cultural life script within the broader standard American one (see Haque \& Hasking, 2010).

Non-native speakers of English also rated their CLS events as occurring at an significantly older age than native speakers $[t(98)=-2.17, S E D=.94]$, driving the significant interaction between native speaking of English and life story event type on what age the event would be expected to occur $\left[F(2,90)=3.54, M S E=23.53, \eta_{p}{ }^{2}=.07\right]$. This may be because they entered the American culture at an older age and therefore experienced the milestones of CLS events later than native speakers typically do, which in turn likely influenced their ratings of expected age for these events (see Figure 2 for expected age for all event categories).

Native speaking of English did not seem to affect how participants rated the importance of events in the different categories. We suspected that this may be due to the small number of non-native speakers of English in the sample, given the trend seen in Figure 3. Thus, we probed further into the relationship between event categories and native speaking of English. Nonnative speakers appear to rate their Unique Life Story events as less important compared to CLS events (5.35 for Unique Life Story events versus 6.20 for CLS events), though with so few participants, the relationship is not significant, $t(18)=1.75, \operatorname{SEM}=.48, p=.098$. Native speakers of English, on the other hand, seem to value the Unique Life Story events that they experience 
as equally as important as CLS events, $t<1$ (the rating of Unique Life Story events does not significantly differ from Non-CLS events, however, $t<1$ ). One possibility is that native speakers feel that the unique life story events in their lives are quite significant in the very fact that they deviate from the cultural life script. It could be the case that this is a finding that would be unique to the American culture as it is quite important to Americans' sense of self that they are individuals, special in his/her own right, and differ from the crowd (Sayre, 2002; Settersten \& Hagestad, 1996). More broadly, there are a number of potential reasons for this trend; one such explanation might be that non-native speakers are highly motivated to fit into their new culture and therefore find their experiences of CLS events to be more momentous in their lives than events that are uniquely in life stories. While native speaking of English affected participants' ratings of the importance of events in their lives, this demographic factor did not affect participants' prevalence or valence ratings. That is, regardless of whether or not they were native speakers of English, participants similarly rated how common life story events were as well as the emotional nature of those events $\left(F_{s}<1\right)$.

We can draw several tentative conclusions from this investigation of the influence of Native English speaking on different aspects of commonly experienced life story events. First, non-native speakers of English seem to have been less influenced by the CLS in their nominations of life story events as they listed many more unique life story events than native speakers. Interestingly, however, these same non-native speakers rated the CLS events that they did include in their life stories as slightly more important than their uniquely experienced life events. Furthermore, an intriguing finding here is that native speakers rated Unique life 
story events as no less important than CLS events, suggesting that these experiences outside the cultural life script are quite important to them.

\section{Future Directions}

While the secondary analyses of the Rubin et al. (2009) American dataset on life script and life story events are informative, they are just the first step toward understanding the relationship between people's most significant personal life stories and what they consider to be the most important events for a typical person in their culture. For example, the nativeness of the language of the society in which one lives seems to affect people's nominations of important life story events as well as their understanding and subscription to the relevant cultural life script. However, before examining the nature of an individual's identification with a culture, we must first understand why some common life story events overlap between personal life stories and cultural life scripts whereas others only appear in personal life stories.

While categorizing events that people commonly include in their life stories may give us an overview of the events (CLS events, Non-CLS events, and Unique Life Story events), the question remains, what specific qualities of these "common" life stories lead some of them to manifest in the cultural life script while others do not? If these two types of events are both commonly experienced by people within a culture, what about Unique Life Story events makes them so? There do appear to be some differences between such events and CLS events and even Non-CLS events as described above. But ratings of prevalence, importance, age, and valence as reported and analyzed here are not enough to understand and characterize the qualitative differences underlying the distinctions between these event types. 
In order to generate what qualities might be relevant, we can look to the definition of the cultural life script. Berntsen and Rubin (2004) were the first to lay out a list of 10 specific attributes of the cultural life script: 1) It consists of semantic knowledge of events that an individual may not have experienced yet, 2) it includes a sequence of temporally ordered events, 3) it is a series of slots and requirements for those slots to be filled with particular events, 4) it forms a hierarchical arrangement with transitional events forming a higher order "scene" within which a number of subordinate events are nested, 5) it is used to process and organize life stories, 6) it consists of culturally important transitional events with culturally sanctioned timing, 7) it is not extracted from personal recurrent experienced but passed on through tradition, 8) it represents an idealized life rather than a typical life, 9) it has an overrepresented number of positive events, and 10) it favors events expected to occur within the time period of the reminiscence bump. Quantifying these attributes across the different categories of commonly experienced life stories may allow us to pinpoint which aspects of the cultural life script are particularly poignant for a life story event to be even considered as part of a culture's proscribed prototypical life.

It is also important to consider the nature of life stories. Habermas and Bluck (2000) provide four types of coherence that define a "good" life story: temporal, biographical, causal, and thematic. Temporal coherence refers to a sense of linear ordering in time of the events in one's life story. Biographical coherence comes from a cultural concept of biography and seems to overlap greatly with the idea of a "cultural life script," citing "the normative cultural notion of the facts and events that should be included in life narratives...defin[ing] conventional life 
phases" (Habermas \& Bluck, 2000). Causal coherence refers to the explanatory nature of the life story, including offering rationale for one's actions and changes in one's values or personality over time. Finally, thematic coherence regards an evaluative component in the life story, interpreting the events in one's life to give them meaning within the context of one's set of life experiences. These forms of coherence help identify what ought to be included when one is telling his/her life story. Though the life story task in Rubin et al. (2009) and many other studies (e.g. Bohn, 2010; Bohn \& Berntsen, 2008, 2011; Berntsen \& Rubin, 2004; Erdogan et al., 2008; Janssen \& Rubin, 2011) does not necessarily lead to participants considering how they would tell their full life story in narrative form, these types of coherence likely influence which events they choose to include among the seven most important events from a lifetime of experiences.

Taking together the characteristics of cultural life scripts and the coherences involved in making a "good" life story, we can begin to systematically distinguish which aspects of a cultural life script and people's individual life stories are integral for whether commonly experienced life events are also part of the cultural life script (CLS events), sometimes thought to be part of it (Non-CLS events), or remain uniquely important to individuals' life stories. For example, through these definitions and other work (Clarke, 1995; Neugarten et al., 1965; Plath \& Ikeda, 1975; Settersten \& Hagestad, 1996), we can note that the timing of events, even culturally sanctioned ones, is key. Researchers even suggest that when asked to recall a life story event, people are likely to consider when their culture (and thus, cultural life script) proscribes the event to occur as a reference point for the memory (Berntsen \& Rubin, 2004; Haque \& Hasking, 
2010). Some events are likely to be more time-locked than others; by time-locked, we mean that a given event is expected to occur within a particular time window and that it is only appropriate or culturally acceptable during that time frame. Thus, this is one potential factor that distinguishes the different types of commonly experienced life story events: CLS events could tend to be more time-locked while events that are commonly experienced but uniquely in life stories could more easily happen at any time. Similarly, there are many other characteristics of commonly experienced life events that could help to better define what sorts of experiences are likely to remain uniquely part of people's life stories and the role of these events in people's conceptions of their own lives.

\section{Summary and Conclusions}

People show a high level of agreement as to which events belong in the cultural life script. However, at the same time, many events that they deem as important to their individual life stories are not part of the life script. Perhaps even more interestingly, many of these unique life story events are widely shared across individuals, as we have shown here. This shows that individual life stories have more in common that what the life script prescribes. More research is needed to disentangle exactly which factors are decisive for common life events to be either included in or excluded from the normative life script. Here we have taken a first step. We also began the exploration of the role of diversity within a given culture through differences between native and non-native speakers of English in the present sample. We found that the non-native speakers had more unique life story events than the native speakers, which may suggest that the former is less well integrated in the overarching standard American culture. In 
a wider context, this type of analysis may be promising as a method for gaining more finegrained insights into how much concrete life events as experienced and interpreted within certain subcultures (including regional, gender-based, and ethnic groups) deviate from the life script of the overarching culture. Identifying specific life events that are central in the individual life stories in such social groups while of little centrality in the dominant life script of the culture may help in identifying potential points of conflict and thereby ease the integration and mutual understanding across different social groups. 


\section{References}

Bernsten, D., \& Rubin, D.C. (2002). Emotionally charged autobiographical memories across the life span: The recall of happy, sad, traumatic and involuntary memories. Psychology and Aging, 17(4), 636-652.

Berntsen, D., \& Rubin, D. C. (2004). Cultural life scripts structure recall from autobiographical memory. Memory \& Cognition, 32(3), 427-442.

Bohn, A. (2010). Generational differences in cultural life scripts and life story memories of younger and older adults. Applied Cognitive Psychology, 24, 1324-1345.

Bohn, A., \& Berntsen, D. (2008). Life story development in childhood: The development of life story abilities and the acquisition of cultural life scripts from late middle childhood to adolescence. Developmental Psychology, 44(4), 1135-1147.

Bohn, A., \& Berntsen, D. (2011). The reminiscence bump reconsidered: Children's prospective life stories show a bump in young adulthood. Psychological Science, 22(2), 197-202.

Clarke, D. D. (1995). Life scripts: Implicit representations of life-course patterns. Journal of Social Behavior and Personality, 10(4), 871-884.

Collins, K. A., Pillemer, D. B., Ivcevic, Z., \& Gooze, R. A. (2007). Cultural scripts guide recall of intensely positive life events. Memory \& Cognition, 35(4), 651-659.

Conway, M. A., \& Bekerian, D. A. (1987). Organization in autobiographical memory. Memory \& Cognition, 15(2), 119-132.

Erdoğan, A., Baran, B., Avlar, B., Taş, A. C., \& Tekcan, A. I. (2008). On the persistence of 
positive events in life scripts. Applied Cognitive Psychology, 22, 95-11).

Glück, J. \& Bluck, S. (2007). Looking back across the life span: A life story account of the reminiscence bump. Memory \& Cognition, 35(8), 1928-1939.

Haque, S. \& Hasking, P. A. (2010). Life scripts for emotionally charged autobiographical memories: A cultural explanation of the reminiscence bump. Memory, 18(7), 712-729.

Habermas, T., \& Bluck, S. (2000). Getting a life: The emergence of the life story in adolescence. Psychological Bulletin, 126, 748-769.

Janssen, S. M. J., \& Rubin, D. C. (2011). Age effects in cultural life scripts. Applied Cognitive Psychology, 25, 291-298.

Luborsky, M. R. (1993). The romance with personal meaning in gerontology: Cultural aspects of life themes. The Gerontologist, 33, 445-452.

McAdams, D. P. (2001). The psychology of life stories. Review of General Psychology, 5(2), $100-122$

Neugarten, B. L., Moore, J. W., \& Lowe, J. C. (1965). Age norms, age constraints, and adult socialization. American Journal of Sociology, 70(6), 710-717.

Plath, D. W., \& Ikeda, K. (1975). After coming of age: Adult awareness of age norms. Socialization and Communication in Primary Groups.

Rubin, D. C., \& Berntsen, D. (2003). Life scripts help to maintain autobiographical memories of highly positive, but not highly negative, events. Memory \& Cognition, 31(1), 1-14.

Rubin, D. C., Bertnsen, D., \& Hutson, M. (2009). The normative and the personal life: Individual differences in life scripts and life story events among USA and Danish undergraduates. 
Memory, 17(1), 54-68.

Sayr, J. (2002). Personal narratives in the life stories of older adults. Journal of Geriatric Psychiatry, 35(2), 125-150.

Settersten, R. A., Jr., \& Hagestad, G. O. (1996). What's the latest? Cultural age deadlines for family transitions. The Gerontologist, 36, 178-188.

Thomsen, D. K., \& Bernsten, D. (2008). The cultural script and life story chapters contribute to the reminiscence bump. Memory, 16(4), 420-435.

Tomasello, M. (2001). Cultural transmission: A view from chimpanzees and human infants. Journal of Cross-Cultural Psychology, 32, 135-146.

Tulving, E. (1985). How many memory systems are there? American Psychologist, 40 (4), 385398. 


\section{Author's Note}

The first author is supported by a National Science Foundation Graduate Research Fellowship, and the present investigation was supported by the National Science Foundation Nordic Research Opportunity awarded to the first author. This work was also supported by the Danish National Research Foundation and the Danish Council for Independent Research: Humanities. We would like to thank the Center on Autobiographical Memory Research for their comments regarding the theoretical questions addressed here. 
Table 1. Categories of Commonly Nominated Life Story Events

\begin{tabular}{|c|c|c|}
\hline Event Type & Life Script & Examples \\
\hline $\begin{array}{l}\text { Cultural Life } \\
\text { Script Event } \\
\text { (CLS event) }\end{array}$ & $\begin{array}{l}\text { Present in the Life script } \\
\text { (nominated in the life script } \\
\text { task by } 4 \text { or more people) }\end{array}$ & $\begin{array}{l}\text { college, other's death, high school, } \\
\text { leaving home }\end{array}$ \\
\hline $\begin{array}{l}\text { Non-Cultural } \\
\text { Life Script Event } \\
\text { (Non-CLS event) }\end{array}$ & $\begin{array}{l}\text { Nominated in the life script } \\
\text { task by more than } 1 \text {, but less } \\
\text { than } 4 \text { people }\end{array}$ & $\begin{array}{l}\text { Long trip, having siblings, } \\
\text { accident/injury, learning to read/write }\end{array}$ \\
\hline $\begin{array}{l}\text { Unique Life } \\
\text { Story Event }\end{array}$ & $\begin{array}{l}\text { Never nominated in the life } \\
\text { script task }\end{array}$ & $\begin{array}{c}\text { Moving, playing a sport, playing an } \\
\text { instrument, having an } \\
\text { epiphany/realization }\end{array}$ \\
\hline
\end{tabular}


Table 2. Frequency of Common Life Story Events in the Life Script and in Life Stories

\begin{tabular}{|c|c|c|c|}
\hline Event Type & Event & $\begin{array}{c}\text { Frequency of } \\
\text { Nomination in } \\
\text { Life Script Events }\end{array}$ & $\begin{array}{c}\text { Frequency of } \\
\text { Nomination in } \\
\text { Life Stories }\end{array}$ \\
\hline \multirow{15}{*}{$\begin{array}{l}\text { Cultural Life } \\
\text { Script Events }\end{array}$} & College & 54 & 86 \\
\hline & Begin School & 48 & 20 \\
\hline & High school & 46 & 60 \\
\hline & First Job & 38 & 5 \\
\hline & Begin Talking & 29 & 7 \\
\hline & Begin Walking & 27 & 7 \\
\hline & Go to School & 22 & 20 \\
\hline & Other's Death & 20 & 32 \\
\hline & Own Birth & 18 & 13 \\
\hline & Fall in Love & 14 & 26 \\
\hline & Begin Driving & 13 & 13 \\
\hline & First Sex & 9 & 8 \\
\hline & Leave Home & 8 & 10 \\
\hline & Begin Daycare & 5 & 4 \\
\hline & First Kiss & 4 & 7 \\
\hline \multirow{12}{*}{$\begin{array}{l}\text { Non-Cultural } \\
\text { Life Script } \\
\text { Events }\end{array}$} & Having Siblings & 3 & 23 \\
\hline & Accident/Injury & 3 & 10 \\
\hline & Learning to Read/Write & 3 & 5 \\
\hline & Long Trip & 2 & 20 \\
\hline & Events with Family & 2 & 5 \\
\hline & Having Peers & 2 & 5 \\
\hline & Serious Disease & 2 & 5 \\
\hline & Develop a Belief System & 1 & 9 \\
\hline & Parent's Divorce & 1 & 9 \\
\hline & Meeting a True Friend & 1 & 5 \\
\hline & Earn First Money & 1 & 4 \\
\hline & Fights involving Parents & 1 & 4 \\
\hline \multirow{11}{*}{$\begin{array}{l}\text { Unique Life } \\
\text { Story Events }\end{array}$} & Moving & 0 & 42 \\
\hline & Major Achievement & 0 & 38 \\
\hline & Playing a Sport & 0 & 22 \\
\hline & Playing an Instrument & 0 & 11 \\
\hline & Bar/Bat Mitzvah & 0 & 8 \\
\hline & $\begin{array}{l}\text { Meeting Current Significant } \\
\text { Other }\end{array}$ & 0 & 8 \\
\hline & Long-term Relationship & 0 & 8 \\
\hline & Living in Another Country & 0 & 6 \\
\hline & Parents (re)marry & 0 & 5 \\
\hline & Having an Epiphany/Realization & 0 & 4 \\
\hline & Vacation & 0 & 5 \\
\hline
\end{tabular}


Table 3. Nominations and Ratings of Commonly Experienced Life Story Events

\begin{tabular}{|c|c|c|c|}
\hline & $\underline{\text { CLS Events }}$ & $\underline{\text { Non-CLS Events }}$ & $\underline{\text { Unique Events }}$ \\
\hline Number of Events & 3.18 & .77 & 1.58 \\
\hline Prevalence of Events & 64.43 & 45.96 & 40.00 \\
\hline Importance of Events & 6.07 & 5.87 & 5.78 \\
\hline Valence of Events & 1.36 & .88 & 1.70 \\
\hline
\end{tabular}

Note: The number of events is out of a total of 7 nominated in the life story task overall; the prevalence ratings were on a scale of 1 to 100; the importance ratings were on a scale of 1 to 7 ; and valence was rated from -3 to +3 . 
Figure 1. Number of Nominated Commonly Experienced Life Story Events based on Event Type and Native Speaking of English

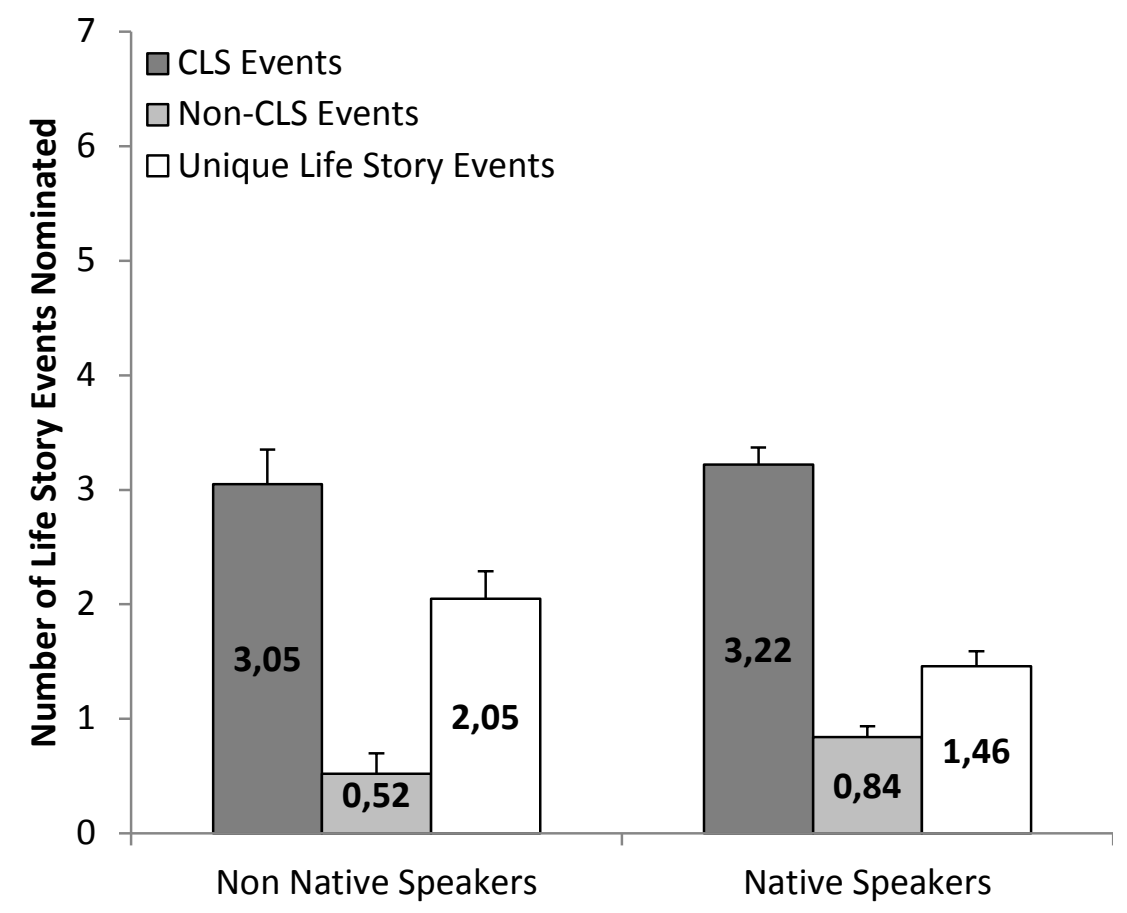


Figure 2: Age Ratings of Nominated Commonly Experienced Life Story Events based on Event

Type and Native Speaking of English

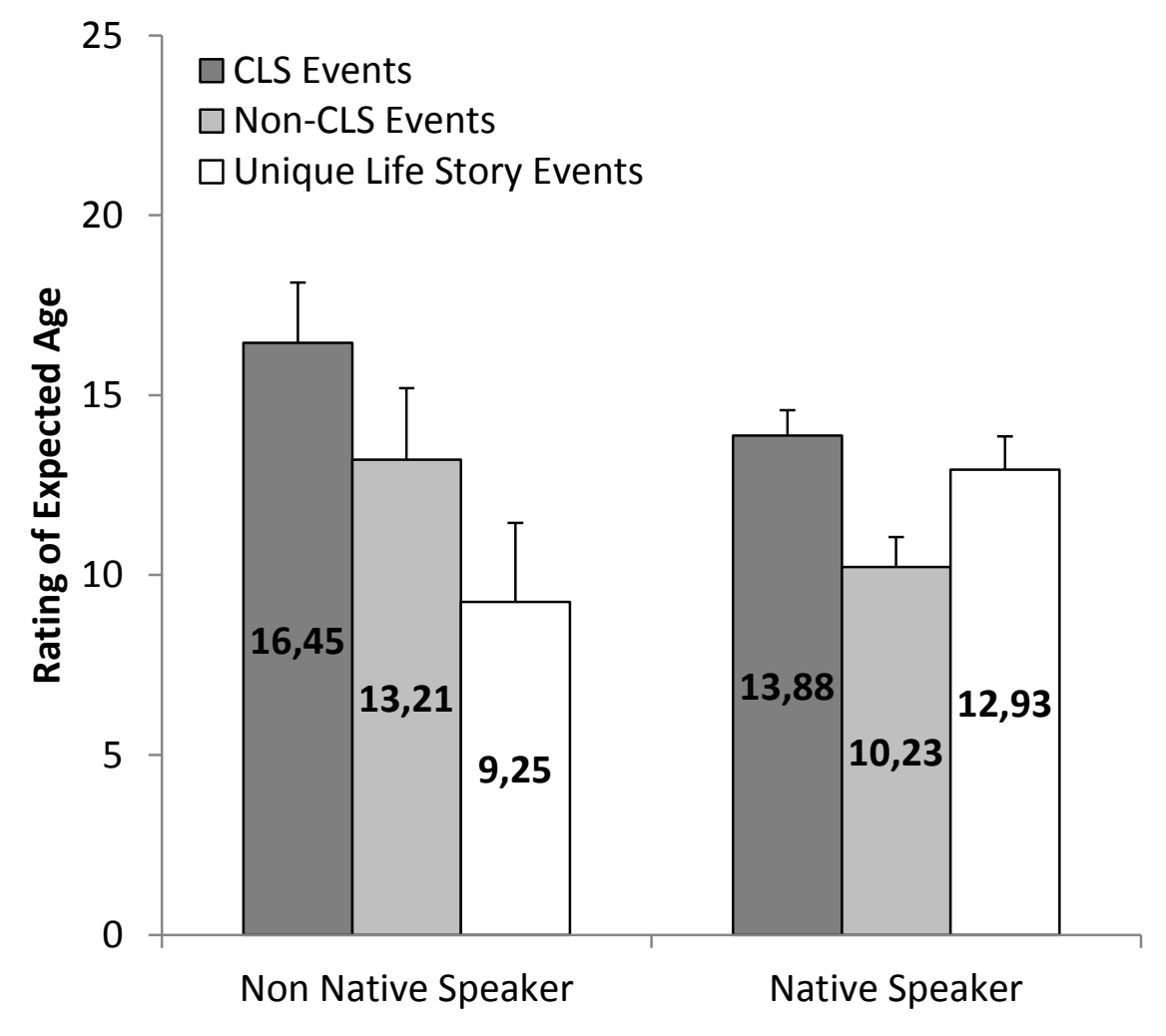


Figure 3: Importance Ratings of Nominated Commonly Experienced Life Story Events based on

Event Type and Native Speaking of English

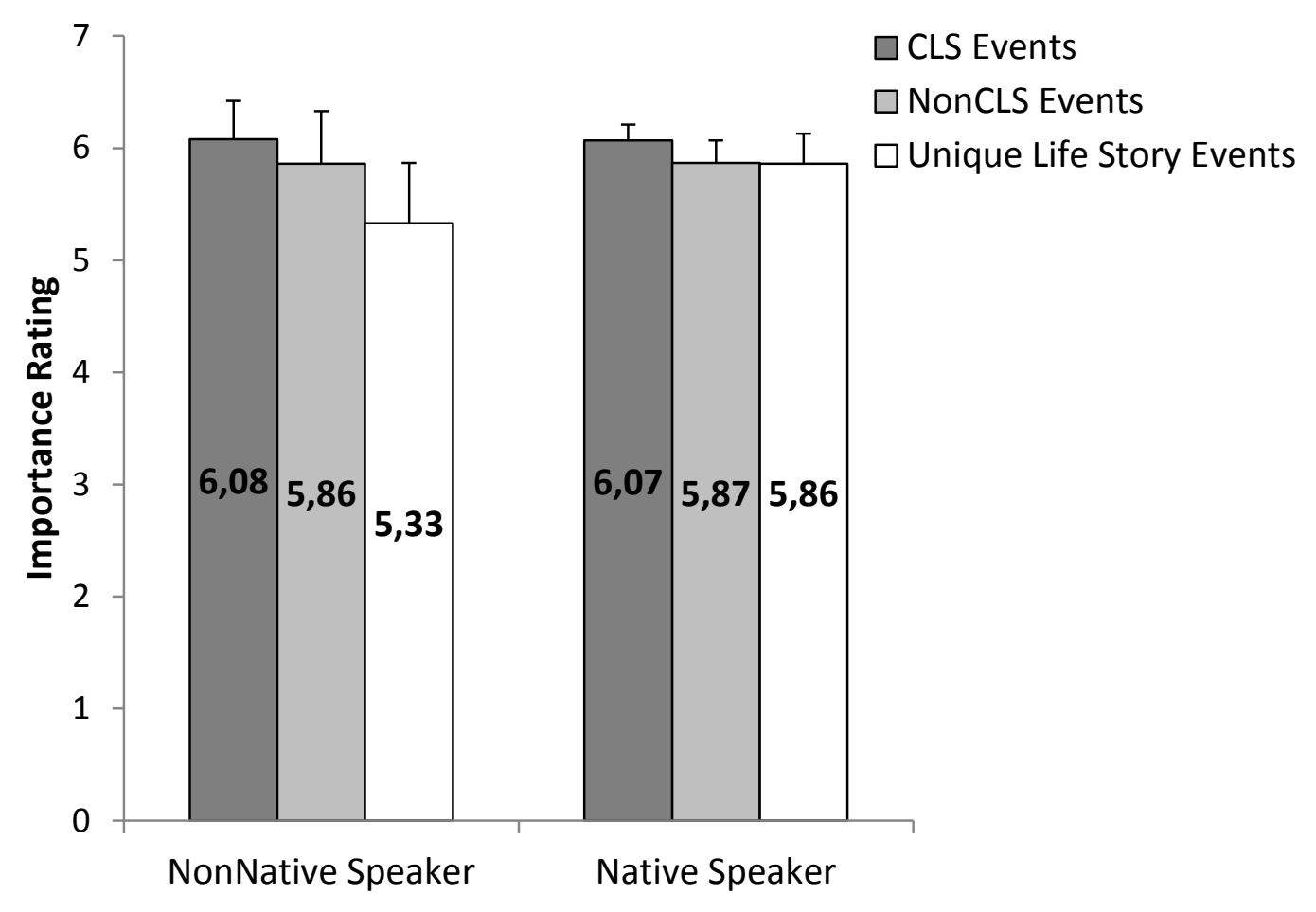

